\title{
Characterization of Screw Dislocations in a 4H-silicon Carbide Diode using X-ray Microbeam Three-dimensional Topography
}

\author{
R. Tanuma ${ }^{1-2, a}$, T. Tamori $i^{1-2, b}, Y$. Yonezawa ${ }^{1-2, c}$, \\ H. Yamaguchi ${ }^{3, \mathrm{~d}}$, H. Matsuhata ${ }^{3, \mathrm{e}}$, K. Fukuda ${ }^{3, \mathrm{f}}$, and K. Arai ${ }^{3, g}$
}

${ }^{1}$ Fuji Electric Device Technology Co., Ltd., 4-18-1 Tsukama, Matsumoto, Nagano 390-0821, Japan

${ }^{2}$ Research and Development Association for Future Electron Devices, AIST Tsukuba Central 2, 1-1-1 Umezono, Tsukuba, Ibaraki 305-8568, Japan

${ }^{3}$ National Institute of Advanced Industrial Science and Technology, AIST Tsukuba Central 2, 1-1-1 Umezono, Tsukuba, Ibaraki 305-8568, Japan

atanuma-ryohei@fujielectric.co.jp, ${ }^{b}$ tamori-tae@fujielectric.co.jp, cyonezawa-yoshiyuki@fujielectric.co.jp, dyamaguchi-hr@aist.go.jp, ${ }^{\text {}}$ h.matsuhata@aist.go.jp, fk-fukuda@aist.go.jp, ${ }^{9}$ arai-kazuo@aist.go.jp

Keywords: screw dislocation, strain, microplasma, leak, X-ray, microbeam, topography, V-slit

\begin{abstract}
This paper describes the study of non-hollow-core elementary screw dislocations (SDs) in silicon carbide ( $\mathrm{SiC}$ ) diodes using X-ray microbeam three-dimensional topography. Strain analysis shows that typical screw dislocations having a symmetric strain field tend to cause microplasma breakdown, whereas deformed SDs do not. The symmetry break in SDs will relax the focussing of strain and lessen the formation of defects, thereby leading to the desirable non-leak property.
\end{abstract}

\section{Introduction}

Among the structural defects in silicon carbide (SiC), harmful micropipes have been significantly decreased in density, and focus is now shifting toward non-hollow-core elementary screw dislocations (SDs) that are responsible for microplasma breakdown (MB) in a reverse-biased condition [1]. MB is usually explained as a result of the local reduction of band gap and/or the presence of recombination centers along a dislocation line [1]. However, the mechanism of MB is not fully understood, such as the largely different current-leak levels among similar SDs. In order to better understand the MB phenomena, we conducted crystallographic analysis of SDs in SiC diodes using $\mathrm{X}$-ray microbeam three-dimensional (3D) topography that we have developed [2]. Unlike conventional methods, 3D topography can provide highly depth-resolved strain maps in various cross sections of semiconductor materials. This paper describes the strain analysis of ten SDs found in the diodes, half of which were accompanied by MB. The strain analysis shows that a symmetry break in SDs leads to the non-leak property.

\section{Experimental Method}

Two SiC p-n diodes (D1 and D2) fabricated on the carbon face of an $8^{\circ}$-off-cut $\{0001\} 4 \mathrm{H}-\mathrm{SiC}$ wafer were examined as samples. The diodes consist of $\mathrm{n}$ (net donor concentration: $3-4 \times 10^{17} \mathrm{~cm}^{-3}$ ) and $\mathrm{p}^{+}$epitaxial layers 3.1- $\mu \mathrm{m}$ and 1.4- $\mu \mathrm{m}$-thick, respectively, with ideal blocking voltages of 120 to $130 \mathrm{~V}$. The plane areas of D1 and D2 are $1.5 \times 1.5 \mathrm{~mm}^{2}$ and $1 \times 1 \mathrm{~mm}^{2}$, respectively. Prior to X-ray analysis, we performed electroluminescence (EL) tests just

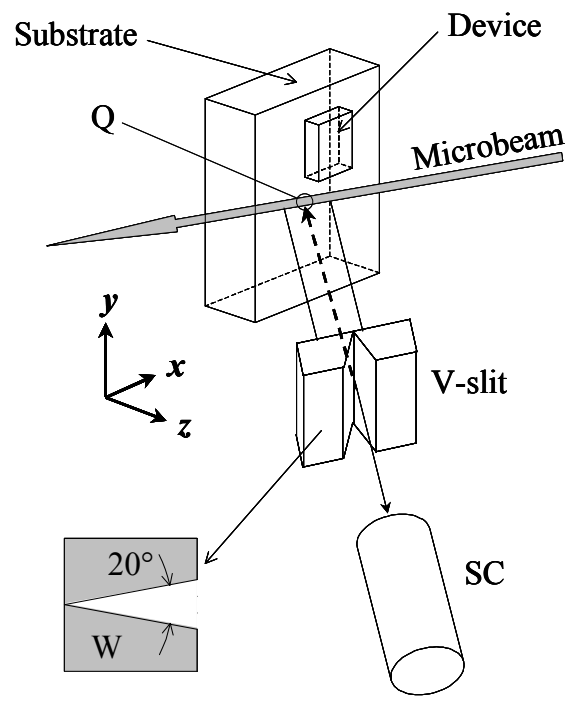

Fig. 1. Microbeam X-ray diffraction with the $\mathrm{V}$-slit. The V-slit is a metal contact made of tungsten (W) with polished slanted edges. 
(a)

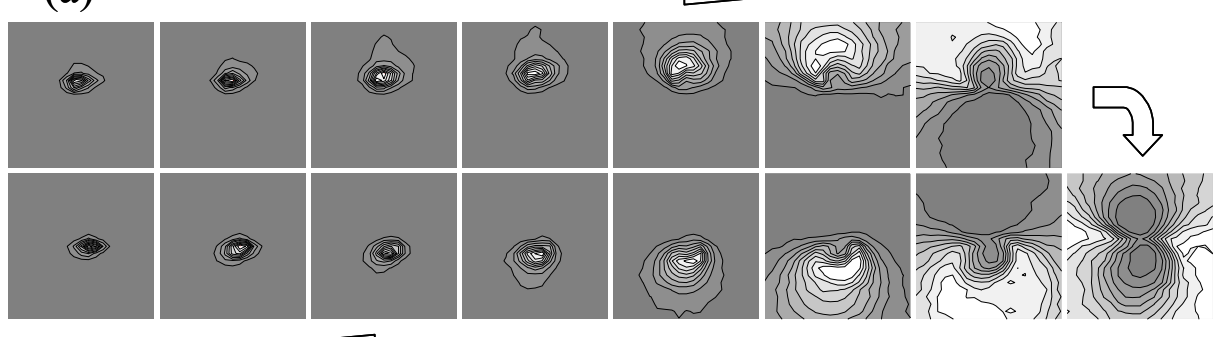

(b)

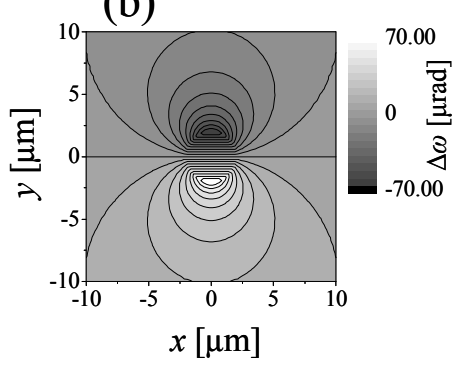

(c)
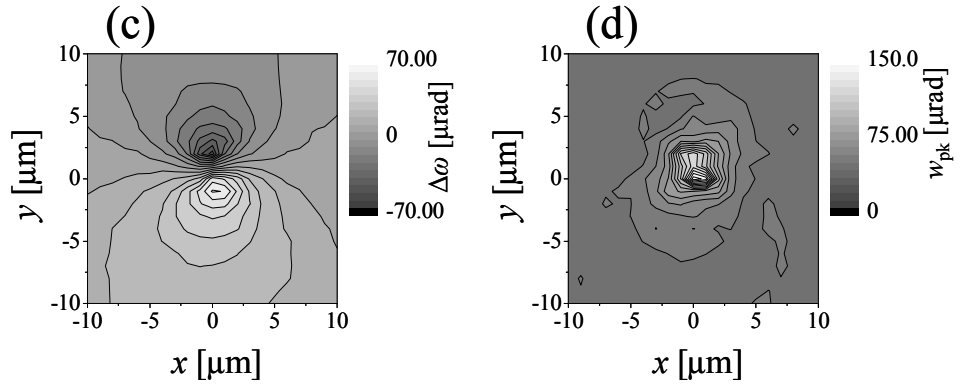

Fig. 2. Example of strain analysis of SD. We obtained 15 topographs at consecutive $\omega$ angles in steps of $14 \mu \mathrm{rad}$. The topographic data were converted into rocking curves, and peak shifts $(\Delta \omega)$ and peak widths $\left(w_{\mathrm{pk}}\right)$ were estimated at each sampling position [(c), (d)]. The calculated $\Delta \omega$ map (b) is in good agreement with the observed one (c). The radius of the core $\left(R_{\text {core }}\right)$ is about $2 \mu \mathrm{m}$, and $w_{\mathrm{pk}}$ is at least two times larger in the core than in the periphery $(\mathrm{d})$.

below the breakdown voltages to find the MB positions; the reverse voltages (currents) were $90 \mathrm{~V}$ ( 2 $\mu \mathrm{A})$ and $70 \mathrm{~V}(5 \mu \mathrm{A})$ for $\mathrm{D} 1$ and $\mathrm{D} 2$, respectively.

X-ray measurements were conducted on the beam line BL24XU at SPring-8 [3]. An undulator-radiated X-ray beam $(E=15 \mathrm{keV})$ was focused on a sample by using a bent cylindrical mirror with a focal length of about $1 \mathrm{~m}$. The full widths at half maximum (FWHM) of the focused beam were $1.7 \mu \mathrm{m}$ and $0.8 \mu \mathrm{m}$ in the horizontal and vertical directions, respectively, and the beam divergence was $23 \mu \mathrm{rad}$. The photon flux was approximately $5 \times 10^{6}$ photons/s on the samples.

We used a special slit with a V-shaped crevice (V-slit) in microbeam X-ray diffraction (Fig. 1). The V-slit is a metal contact made of tungsten (W) with polished slanted edges and an opening angle of $20^{\circ}$ [2]. We used Bragg-case geometry with 0016 reflection, in which the scattering vector is parallel to the $c$-axis. The diffraction beam impinges on the $\mathrm{V}$-slit, through which a thin beam passes to reach a YAP scintillation counter (SC). We can thus observe a small region $\mathrm{Q}$ where the incident beam crosses the extended line (broken arrow) of the beam passing through the slit, and scanning the sample position provides 3D topography. Using this method, we can obtain a map of strain from consecutive diffraction images at step-scanned rocking angles $(\omega)$.

\section{Results and Discussion}

Strain Map. Figure 2 shows an example of strain analysis for SD [No. 5(LL) described later]. As shown in Fig. 2a, we obtained 15 diffraction images (topographs) at consecutive $\omega$ angles in steps of $14 \mu \mathrm{rad}$ on a plane $2-\mu \mathrm{m}$ deep from a device surface (near the $\mathrm{p}-\mathrm{n}$ junction). The topographs were $20 \times 20 \mu \mathrm{m}^{2}$ in size with a sampling

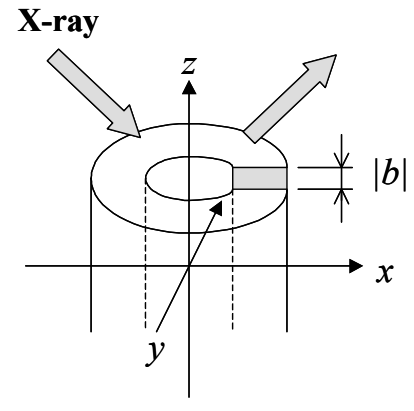

Fig. 3. Model of a screw dislocation and coordinate system. step of $1 \mu \mathrm{m}$. The topograph data were converted into rocking curves at each sampling position, and peak shifts $(\Delta \omega)$ and peak widths $\left(w_{\mathrm{pk}}\right)$ were estimated as shown in Fig. $2 \mathrm{c}$ and $2 \mathrm{~d}$, respectively, by means of Gaussian fitting.

Consider a pure screw dislocation parallel to the $c$-axis lying along the $z$-axis in the coordinate system shown in Fig. 3. This figure illustrates a left-turn SD (step down with a left turn). The strain 
involved is shear strain (lattice tilt), and its $z x$ component is given by $\partial u_{z} / \partial x=(b / 2 \pi) y /\left(x^{2}+y^{2}\right)$, where $u_{z}$ denotes displacement along the $z$-axis, and $b$ the magnitude of the Burgers vector $(>0$ for the left
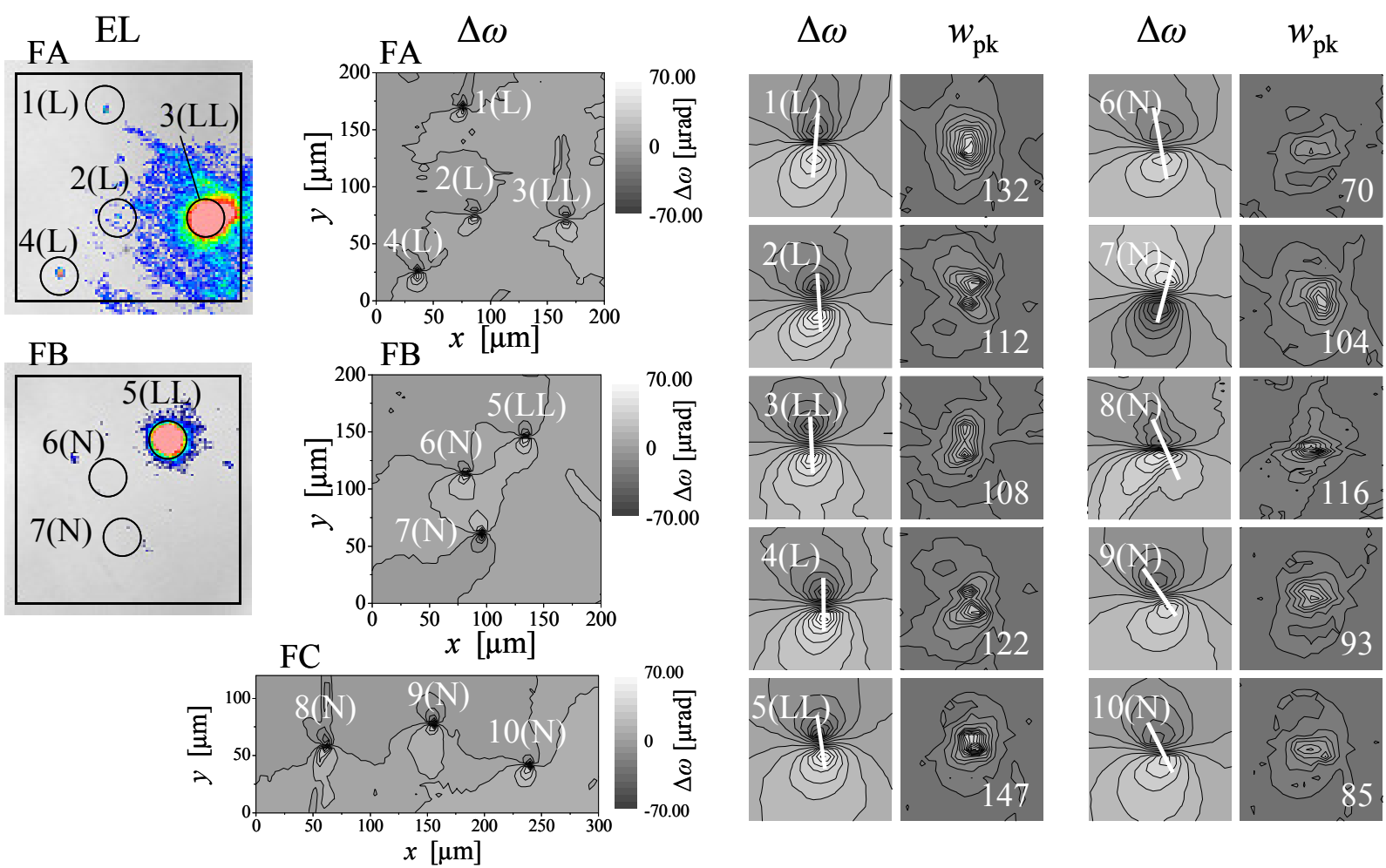

Fig. 4. Results of strain analysis of SDs found in the diodes. In comparing the EL images (at the far left) and the $\Delta \omega$ maps ( in the second column), we classified the SDs into types LL, L and N, standing for "Large Leak," "Leak" and "No leak," respectively. The four columns on the right show the fine maps of $\Delta \omega$ and $w_{\mathrm{pk}}$. The min-max lines (white lines) are almost vertical for the LL and L types, whereas those for the $\mathrm{N}$ type are rotated. The maximum $w_{\mathrm{pk}}$ values indicated in the $w_{\mathrm{pk}}$ maps are smaller for the $\mathrm{N}$ type than for the LL and $\mathrm{L}$ types.

turn). The peak shift is related to the shear strain as $\Delta \omega=-\partial u_{z} / \partial x$. The SD examined is of a left turn because $\Delta \omega<0$ for $y>0$ (Fig. 2c). Figure 2b is $\Delta \omega$ calculated with $b=8.5 \AA$, which is estimated using the method described later. In this calculation, the denominator is constant for $x^{2}+y^{2}<2 \mu \mathrm{m}$ to avoid infinity. The model map (Fig. 2b) well expresses the observed strain field (Fig. 2c), showing that the SD examined is an elementary one, though the $b$ value estimated is a little smaller than $c_{0}=10.06 \AA$ (c-axis lattice constant of $4 \mathrm{H}-\mathrm{SiC}$ ).

We define the core of SD as the region in which the strain exceeds the limit of elasticity, causing plastic deformation (crystal defects), which results in an increase of $w_{\mathrm{pk}}$ as well as the deviation of strain from the SD model. Figure $2 \mathrm{~d}$ shows that the radius of the core $\left(R_{\text {core }}\right)$ is about $2 \mu \mathrm{m}$, and that $w_{\mathrm{pk}}$ is at least two times larger in the core than in the periphery.

Strain and EL Emission. We conducted the strain analysis for SDs found in the diodes (Fig. 4). The regions examined were the flames FA(D1), FB(D2) and FC(D2). The strain maps were obtained with $4-\mu \mathrm{m}$ steps in these flames (in the second column), which include ten SDs numbered 1 to 10. The EL emission images shown at the far left are for FA and FB (with no emission for FC). In comparing these EL images and strain maps, we classified the SDs into types LL, L and N, standing for "Large Leak", "Leak", and "No leak", respectively.

The four columns on the right show the fine maps (in 1- $\mu \mathrm{m}$ steps) of $\Delta \omega$ and $w_{\mathrm{pk}}$ for each SD. Their scales are the same as those shown in Figs. $2 \mathrm{c}$ and $2 \mathrm{~d}$. We can see that only $7(\mathrm{~N})$ is a right-turn SD, and the others are of a left turn. By using the fine $\Delta \omega$ map data, with the core-region data excluded, we can estimate $b$ from the linear fitting of $\Delta \omega$ vs. $y /\left(x^{2}+y^{2}\right)$. Figure 5 shows the estimates of $b, b$ est, obtained with different data-exclusion radiuses $\left(R_{\mathrm{ex}}\right)$. The $b_{\text {est }}$ values are almost constant for $R_{\mathrm{ex}}>2$ $\mu \mathrm{m}$ and are close to $\pm c_{0}$, showing that $R_{\text {core }} \approx 2 \mu \mathrm{m}$, and that all SDs found here are elementary ones. 
In Fig. 4, the white lines in the fine $\Delta \omega$ maps run through the points of positive and negative peaks, and the values in the $w_{\mathrm{pk}}$ maps indicate the maximums of $w_{\mathrm{pk}}$ in $\mu \mathrm{rad}$ units. Note that the min-max lines are almost vertical for the LL and L types, but are rotated for the $\mathrm{N}$ type. The maximum $w_{\mathrm{pk}}$ values are compared in Fig. 6, with an average of $124.2 \mu \mathrm{rad}$ for the LL and L types, and 93.6 $\mu \mathrm{rad}$ for the $\mathrm{N}$ type. The average-difference test (t-test) indicates that the former is larger than the latter with a significance level of $1 \%$.

Thus, $\Delta \omega$ and $w_{\mathrm{pk}}$ are correlated to the extent of current leakage. The strain of SD having typical symmetric strain field steeply increases toward infinity at the center. In N-type SDs, however, the breaking symmetry (tilt of the min-max lines) will relax the focussing of strain and lessen the formation of defects (decrease in $w_{\mathrm{pk}}$ ), thereby leading to the desirable non-leak property. We consider that crystal defects not only cause an increase in recombination centers, but also cause band-gap reduction in $4 \mathrm{H}-\mathrm{SiC}$ because its ideal stacking structure is essential for a wide band gap. The above results support the band-gap reduction and/or recombination centers as the main factors governing the cause of MB.

The mechanism of the symmetry break in SDs is still unclear. We need to clarify whether asymmetrical SDs are generated during epilayer growth, or replicated from the substrate. Another important point is the difference between the LL- and L-type SDs. It seems that the LL phenomena are caused by epilayer defects overlapping with the SD region.

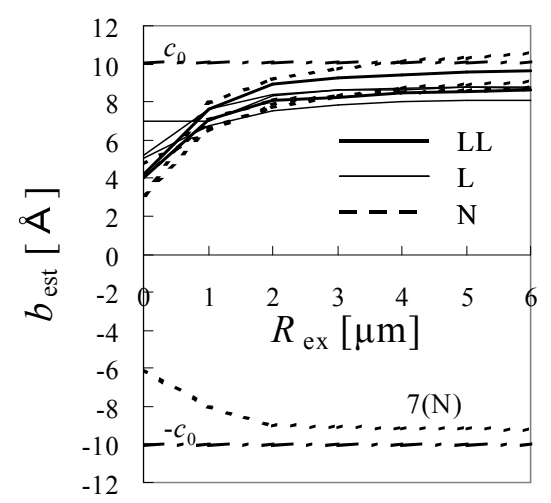

Fig. 5. Plot of $b_{\text {est }}$ Vs. $R_{\text {ex }}$. Linear fitting of $\Delta \omega$ vs. $y /\left(x^{2}+y^{2}\right)$ provides constant $b_{\text {est }}$ if $R_{\text {ex }}>R_{\text {core. The }}$ $b_{\text {est }}$ values for $R_{\text {ex }}>2 \mu \mathrm{m}$ are close to $\pm c_{0}$, showing that $R_{\text {core }} \approx 2 \mu \mathrm{m}$, and the SDs examined are all elementary SDs.

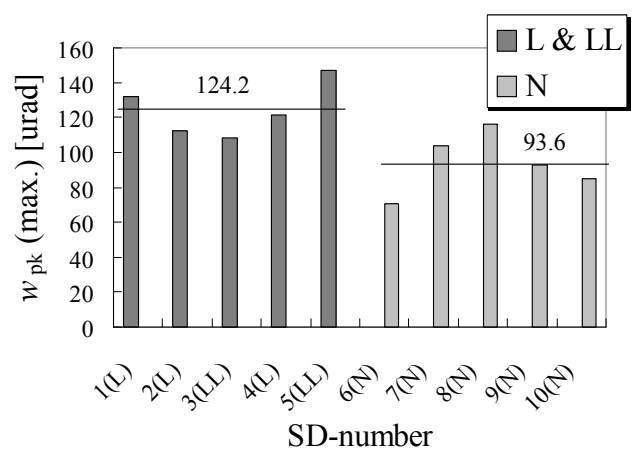

Fig. 6. Comparison of maximum $w_{\mathrm{pk}}$. The t-test indicates that the average of maximum $w_{\mathrm{pk}}$ for the $\mathrm{N}$ types are smaller than that for the LL and $\mathrm{L}$ types with a significance level of $1 \%$.

\section{Summary}

We investigated SDs in $4 \mathrm{H}-\mathrm{SiC}$ diodes using microbeam X-ray 3D topography. From the strain analysis of SDs with varying current-leak levels, we found that leaky SDs have a symmetric strain field, whereas symmetry is broken in SDs without current leak. The symmetry break will relax the focussing of strain and lessen the formation of defects, thereby leading to the desirable non-leak property.

Acknowledgements. We would like to thank Assoc. Prof. Y. Tsusaka of the University of Hyogo for valuable discussions and fine tuning of the X-ray microbeam. This work was conducted as part of research proposal numbers 2007B3211 and 2008A3211 of SPring-8. This work was partially supported by the NEDO project, Development of Inverter Systems for Power Electronics.

\section{References}

[1] U. Zimmermann, J. Österman, D. Kuylenstierna, A. Hallén, A. O. Konstantinov, W. M. Vetter, and M. Dudley: J. Appl. Phys. Vol. 93 (2003), p. 611

[2] R. Tanuma, T. Kubo, F. Togoh, T. Tawara, A. Saito, K. Fukuda, K. Hayashi, and Y. Tsusaka: phys. stat. sol. (a) Vol. 204 (2007), p. 2706

[3] S. Takeda, K. Yokoyama, Y. Tsusaka, Y. Kagoshima, J. Matsui, and A. Ogura; J. Synchrotron Radiat. Vol. 13 (2006), p. 373 